\title{
Children's vision screening: impact on inequalities in central England
}

\author{
Lucy K Smith, John R Thompson, Geoffrey Woodruff
}

\begin{abstract}
Study objective - To investigate the relationship between age at presentation of amblyopia and social deprivation before and after the introduction of changes to a vision screening service.
\end{abstract}

Design - Two cohorts of children treated for amblyopia in 1983 and 1992.

Setting - The orthoptic department of Leicester Royal Infirmary.

Participants - The 209 patients treated for amblyopia who first attended the orthoptic department in 1983, and 203 who first attended in 1992.

Measurements - Age at presentation to the orthoptic department was the main outcome measure. Social deprivation was measured by Townsend deprivation score for the electoral ward in which the child lived, using 1981 and 1991 census data.

Main results - After the introduction of changes in the screening programme, the mean age at presentation of amblyopia associated with microtropia or no strabismus was reduced from $6 \cdot 6$ years to $5 \cdot 0$ years. In 1983 there was a significant relationship between deprivation and age at presentation $(p=0.0001)$, with those from more deprived areas presenting later. No similar association was found in children referred in $1992(p=0 \cdot 17)$. There was no change in the mean age of presentation of amblyopia associated with a large angle of strabismus (3.3 years in 1983 and 1992) and no relationship between deprivation and age at presentation 1983 or $1992(p=$ 0.24 and $p=0.39$ respectively).

Conclusions - Since the introduction of changes to vision screening, the relationship between social deprivation and the age at presentation of asymptomatic amblyopia seems to have disappeared. Children are now referred earlier and those from deprived areas are not being overlooked.

\section{( $(\mathcal{E}$ Epidemiol Community Health 1995;49:606-609)}

Department of Ophthalmology, University of Leicester, Clinical Sciences Building, Leicester LE2 7LX L K Smith J R Thompson

G Woodruff

Correspondence to: Ms L K Smith.

Accepted for publication May 1995
Amblyopia is the most common visual disability in children, with a prevalence estimated to be between 2 and $5 \% .{ }^{12}$ It is characterised by poor visual acuity in an otherwise healthy eye caused by failure to experience normal vision during early childhood. Misalignment of the eyes (strabismus) and differences in refractive error between the two eyes (anisometropia) may cause a child to use one eye less than the other and so cause amblyopia.
Large angle strabismus in a child is usually noticed by their parents ${ }^{3}$ and when they seek medical help any associated amblyopia is likely to be detected. In contrast, amblyopia associated with a very small angle of strabismus (microtropia) or with anisometropia offers no obvious outward signs and is usually only detected by a vision screening test.

Over the last 20 years a system has been in place in Leicestershire for children to be screened for amblyopia and strabismus. Screening takes place at 6 weeks, $7 \frac{1}{2}$ months, 18 months (since 1991), and $3 \frac{1}{2}$ years. At first, health visitors were responsible for most of the screening and children thought to have strabismus or amblyopia were referred to a consultant ophthalmologist via their GP. Between 1988 and 1991 radical changes were made to the county's vision screening. A major development has been the introduction of a secondary orthoptic screening service which allows a much more prompt and readily available referral service for children suspected of having amblyopia or strabismus at the initial screening. Although most of the initial screening is still carried out by health visitors, responsibility for child health surveillance has been transferred to GPs who are required to make a return for every child screened.

The Hall report ${ }^{4}$ reviewed all screening services for preschool children and highlighted the fact that many new screening programmes were introduced before their benefit had been established. Although the report noted the widespread practice of preschool vision screening, it found no evidence of health gain to support this practice and questioned the continuation of screening tests for amblyopia and strabismus. Orthoptic based preschool screening has been shown to be more effective ${ }^{56}$ but there has been little research to investigate the effectiveness of secondary orthoptic screening.

In a previously reported multicentre study we showed that the age at presentation of children with amblyopia but no strabismus was related to social deprivation ${ }^{7}$ but that there was no similar relationship among children with strabismus. We suggested that this may be because deprivation does not affect the detection of large angle strabismus but does affect a child's access to the screening necessary to detect amblyopia associated with anisometropia or microtropia. This paper investigates the changes in the age of detection of amblyopia over a nine year period during which there have been major changes in the screening services. We hypothesised that among children with microtropia or no strabismus, the introduction of improved referral 
Mean age at presentation (numbers in parenthesis) in relation to the quintile of Townsend deprivation score, year, and type of amblyopia

\begin{tabular}{|c|c|c|c|c|}
\hline \multirow[t]{3}{*}{ Quintile of Townsend deprivation score } & \multicolumn{4}{|c|}{ Type of amblyopia } \\
\hline & \multicolumn{2}{|c|}{ Microtropia or no strabismus } & \multicolumn{2}{|c|}{ Large angle of strabismus } \\
\hline & 1983 & 1992 & 1983 & 1992 \\
\hline $\begin{array}{l}1 \text { Least deprived } \\
2 \\
3 \\
4 \\
5 \text { Most deprived } \\
\text { All } \\
\text { Slope (deprivation) } \\
95 \% \text { confidence interval } \\
\text { p value }\end{array}$ & $\begin{array}{l}5 \cdot 4(10) \\
5 \cdot 4(8) \\
6 \cdot 5(16) \\
7 \cdot 3(17) \\
7 \cdot 1(19) \\
6 \cdot 6(70) \\
0 \cdot 204 \\
0 \cdot 10,0 \cdot 31 \\
0 \cdot 0001\end{array}$ & $\begin{array}{l}4 \cdot 5(17) \\
4 \cdot 8(20) \\
5 \cdot 4(21) \\
5 \cdot 3(20) \\
4 \cdot 7(14) \\
5 \cdot 0(92) \\
0.069 \\
-0 \cdot 03,0 \cdot 17 \\
0 \cdot 17\end{array}$ & $\begin{array}{l}2 \cdot 8(19) \\
3 \cdot 2(28) \\
3 \cdot 3(28) \\
3 \cdot 4(42) \\
3 \cdot 5(22) \\
3 \cdot 3(139) \\
0.051 \\
-0.03,0 \cdot 12 \\
0.24\end{array}$ & $\begin{array}{l}3 \cdot 8(14) \\
3 \cdot 3(18) \\
3 \cdot 3(25) \\
2.9(26) \\
3 \cdot 2(28) \\
3.3(111) \\
-0.041 \\
-0.13,0.05 \\
0.39\end{array}$ \\
\hline
\end{tabular}

processes would lessen or reduce the effect of social deprivation on the age at presentation. We expected no change among children with large angle strabismus.

\section{Methods}

Data were collected on two cohorts of children defined as all children who first attended Leicester Royal Infirmary orthoptic clinic in 1983 or 1992 and who were subsequently treated for amblyopia. Comprehensive information was collected from the orthoptic notes for each patient. This included the type of strabismus, ethnic origin (based on Asian or non-Asian forename ${ }^{8}$ ), postcode, age at presentation, and diagnosis. Large angle strabismus was defined as manifest strabismus on cover testing of more than $5^{\circ}$ and microtropia as strabismus of $5^{\circ}$ or less. Anisometropia was defined as at least one dioptre difference in refraction in either sphere or cylinder between the two eyes. Children who were first treated for amblyopia more than two years after first attending the orthoptic clinic were excluded as being unlikely to have had amblyopia at the time of presentation. To avoid the confounding effect of children attending outside clinics, only children from within a $10 \mathrm{~km}$ radius of the hospital are included in this analysis.

Each child's social deprivation was estimated using the Townsend deprivation score ${ }^{9}$ for the electoral ward in which they lived. This score is calculated using the percentage of households with no car; the percentage of households with more than one person per room; the percentage of households not owner occupied and the percentage of economically active people who are unemployed, as recorded in the census. A 1981 and 1991 computerised postcode directory linked the patient's postcode to the ward in which they lived. Data from the 1981 census were used to calculate the Townsend scores for the 1983 cohort and data from the 1991 census were used for the 1992 cohort. To calculate quintiles of deprivation for each census year we took all the wards in the $10 \mathrm{~km}$ radius and ranked them by their Townsend score. Wards were then placed into five groups containing approximately equal numbers of children, on the basis of the Townsend score, ranging from the least deprived group of wards to the most deprived group of wards.

Linear regression was performed separately for each year and type of strabismus, including the deprivation score as a continuous variable. A model was fitted to the combined data for both years in order to test for an interaction between year and deprivation.

\section{Results}

In 1983, 209 children were treated for amblyopia, of whom 139 had amblyopia associated with a large angle of strabismus and 70 had a microtropia or did not have strabismus at all. In 1992,203 children were treated, 111 with a large angle of strabismus and 92 others. There has thus been a significant change in the proportion of children treated for amblyopia with microtropia or no strabismus from $33 \%$ to $45 \%$ (a change of $12 \%, 95 \%$ confidence interval $(2 \cdot 5 \%, 21 \cdot 2 \%))$.

\section{AMBLYOPIA ASSOCIATED WITH MICROTROPIA OR} NO STRABISMUS

The table shows the mean age at presentation in relation to deprivation, year, and type of amblyopia. Among children with amblyopia associated with microtropia or no strabismus, the mean age of presentation was reduced by 19 months from 6.6 years in 1983 to 5.0 years in 1992 ( $\mathrm{p}=0.0001$ (95\% CI 12.8, 26 months)). The table also shows that in 1983 there was a trend of increasing age of presentation with increasing deprivation but that in 1992 there is no similar pattern. This is illustrated in figures 1 and 2. Fitting a regression model to the data for 1983 shows a significant effect of deprivation on age at presentation $(p=0.0001)$. The model indicates that on average children from the most deprived areas present two and a half years later than those from the least deprived areas. At first sight there seems to be a difference between the age at presentation of Asian children compared with non-Asian children $(p=0.09)$ within the 1983 cohort but this difference is completely explained by adjusting for social deprivation $(p=0.98)$. The same regression fitted to the data for 1992 shows the effects of ethnic origin and deprivation are both non-significant $(p=0.99$ and $\mathrm{p}=0 \cdot 17$ respectively).

The data for the two cohorts were combined in a single regression to test for an interaction between year and deprivation. This interaction was found to approach formal significance $(p=$ 0.066 ) supporting our prior hypothesis of a reduction in the effect of deprivation on age at presentation. 


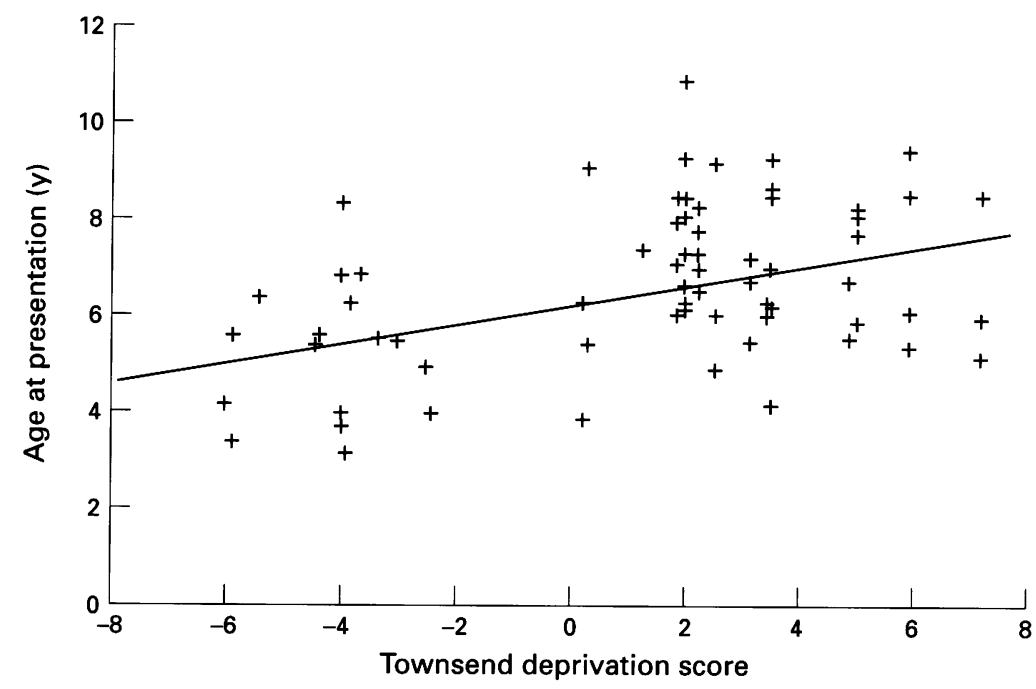

Figure 1 Age at presentation and Townsend deprivation score for children with amblyopia associated with microtropia or no strabismus in 1983

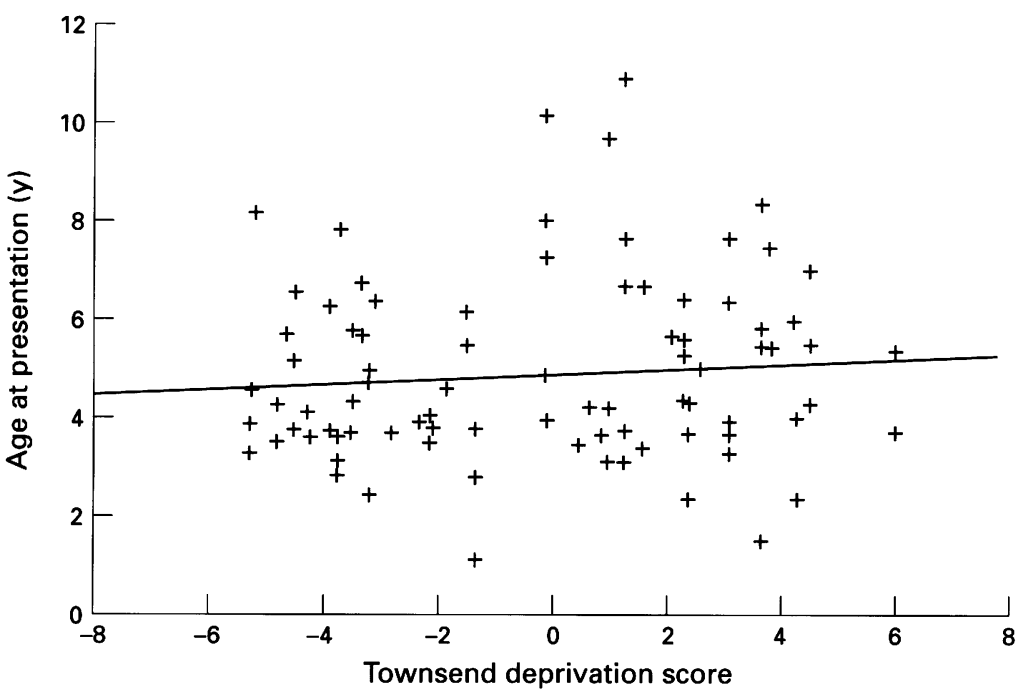

Figure 2 Age at presentation and Townsend deprivation score for children with amblyopia associated with microtropia or no strabismus in 1992.
There have been two changes in the pattern of presentation of amblyopia that is not associated with a large angle of strabismus; the average age has been reduced by 19 months and there is no longer any link between social deprivation and age at presentation.

It is important to consider whether these results could be partly due to the design of our study. By taking children who started treatment in a given year we do not have pure birth cohorts and it is likely that the experience of screening would have varied within the two groups. In particular the changes to child surveillance that were introduced in 1988 were phased in over several years so that some children in the 1992 group may not have experienced the full benefit of the changes. However, this effect would have acted to reduce the apparent impact of the changes to child surveillance. The other problem with the design is that we do not have details of how each child was detected and in particular whether this was through screening or self referral. Thus, we are working with population level information and it is possible, if unlikely, that some external factor may have acted to improve the self referral of children from poorer homes. However, we do know that few children with anisometropic or small angle strabismic amblyopia are detected other than by screening ${ }^{3}$ and we feel confident that any changes to the detection of these conditions would be attributable to changes in screening.

Before 1988 health visitors were required to refer children suspected of a vision problem to their GP who could refer them on to an ophthalmologist. This process offered the opportunity for delay, drop out, and error. The current system whereby children are referred directly from primary screening to the secondary orthoptic screen reduces delay, cuts down the possibility of a child dropping out of the system, and offers a trained assessment of the child's problem.

We believe that improved organisation of child health surveillance is the most likely reason for the removal of the relationship between social deprivation and age at presentation. The percentage of the population of children in Leicestershire screened at $3 \frac{1}{2}$ years of age has increased from $80 \%$ in 1983 to $88 \%$ in 1992 and similarly those screened at $7 \frac{1}{2}$ months has increased from $91 \%$ in 1983 to $95 \%$ in 1992 . An improvement in coverage is likely to have particularly benefited children from poorer areas.

There were a third more cases of amblyopia without a large angle of strabismus treated in 1992 than in 1983, with no change in the size of the population. It is possible that in 1983 a smaller proportion of those detected with amblyopia were treated, or that there has been a rise in the incidence of amblyopia associated with microtropia or anisometropia but we think this is unlikely. Rather we believe that there has been an increase in detection with children being treated today who would previously have gone undetected.

There are no apparent changes in the age at presentation of children with large angle 
strabismus but there has been a reduction in the number of cases treated. It is possible that this is because some children with large angle strabismus are now detected before amblyopia has developed. If this is so screening may actually be lowering the incidence of amblyopia.

Based on our data we estimate that about 40 children born in Leicestershire each year will now have amblyopia associated with microtropia or no strabismus detected that would previously have been missed. In addition those children who are picked up at screening now present on average 19 months earlier than before. Finally there is the possibility that screening may have reduced the number of children with strabismus going on to develop strabismus amblyopia.

We thank Fiona Hiscox and Diane Oliver and the staff of the We thank Fiona Hiscox and Diane Oliver and the staff of the orthoptic clinic at Leicester Royal Infirmary for their help in
this study. We also thank the staff in the Department of General this study. We also thank the staff in the Department of General
Practice at St Mary's College, London for access to the 1981 Practice at St Mary's College, London for access to the 1981
frozen postcode directory. Material from Crown-copyright refrozen postcode directory. Material from Crown-copyright re-
cords has been made available through the Post Office and the
ESRC Data Archive. We gratefully acknowledge the financial support of the British Council for Prevention of Blindness, the Iris fund for Prevention of Blindness, the British Orthoptic Society and the Anne Allerton Fund.

1 Von Noorden GK. Binocular vision and ocular motility: theory and management of strabismus. 4 th ed. St. Louis: Mosby, 1985;208-9.

2 Thompson JR, Woodruff G, Hiscox F, Strong N, Minshull C. The incidence and prevalence of amblyopia detected in C. The incidence and prevalence of amblyo
childhood. Public Health 1991;105:455-63.

3 Wang Yu-dong, Thompson JR, Goulstine DB, Rosenthal AR. A survey of the initial referral of children to an ophthalmology department. Br $\mathcal{F}$ Ophthalmol 1990;74:650-3.

4 Hall DMB ed. Health for all children. Oxford: Oxford University Press, 1989.

5 Bolger PG, Stewart-Brown SL, Newcombe E, Starbuck A. Vision screening in pre-school children: comparison of orthoptists and clinical medical officers as primary screeners. BMF 1991;303:1291-4.

6 Jarvis SN, Tamhne RC, Thompson L, Francis PM. Anderson J, Colver AF. Preschool vision screening. Arch Dis Child 1990;65:288-94.

7 Smith LK, Thompson JR, Woodruff G, Hiscox F, Social deprivation and age at presentation of amblyopia. F Public Health Med 1994;16:348-51.

8 Nicoll A, Bassett K, Ulijaszek SJ. What's in a name? Accuracy of using surnames and forenames in ascribing Asian ethnic identity in English populations. $\mathcal{F}$ Epidemiol Community Health 1986;40:364-8.

9 Townsend P, Phillimore P, Beattie A. Health and deprivation. inequality and the North. London: Croom Helm, 1988. 